\title{
Risk averse submodular utility maximization
}

\author{
Takanori Maehara \\ Department of Mathematical and Systems Engineering, Shizuoka University \\ 3-5-1 Johoku, Naka-ku, Hamamatsu, Shizuoka, 432-8561, Japan
}

\begin{abstract}
In this study, we investigate risk averse solutions to stochastic submodular utility functions. We formulate the problem as a discrete optimization problem of conditional value-at-risk, and prove hardness results for this problem.

Keywords: submodular function, conditional value-at-risk, computational complexity
\end{abstract}

\section{Introduction}

For finite set $V$, set function $f: 2^{V} \rightarrow \mathbb{R}$ is said to be submodular if it satisfies $f(X)+f(Y) \geq f(X \cap Y)+f(X \cup Y)$ for all $X, Y \subseteq V$. A set function $f: 2^{V} \rightarrow \mathbb{R}$ is said to be monotone if $f(X) \leq f(Y)$ for all $X \subseteq Y \subseteq V$. Monotone submodular functions are natural mathematical models of utility functions [1], which can be approximately maximized in polynomial time under several classes of constraints including cardinality constraints [2], matroid constraints [3], and knapsack constraints [4].

Recently, many practical problems have been formulated as a maximization of the expectation of a stochastic submodular function. Let $f: 2^{V} \times \Omega \rightarrow$ $\mathbb{R}$ be a function such that $f(X, \omega)$ is monotone submodular in $X \subseteq V$ and integrable in $\omega \in \Omega$, where $\Omega$ is a probability space. Here, integrable means that the expectation exists and is finite. We consider $f(X, \omega)$ as a utility of $X \subseteq V$ in a stochastic situation $\omega \in \Omega$. The expected utility is then given by $\mathbf{E}[f(X, \omega)]$, where $\mathbf{E}$ denotes expectation with respect to $\Omega$. Then the expected utility maximization problem becomes the following stochastic

Email address: maehara.takanori@shizuoka.ac.jp (Takanori Maehara)

Preprint submitted to Elsevier

August 4, 2015

(C) 2015. This manuscript version is made available under the Elsevier user license http://www.elsevier.com/open-access/userlicense/1.0/ 
optimization problem:

$$
\text { maximize } \quad \mathbf{E}[f(X, \omega)], \quad \text { subject to } \quad X \in \mathcal{I},
$$

where $\mathcal{I} \subseteq 2^{V}$ denotes a set of feasible solutions. Since the sum of monotone submodular functions is also monotone submodular, problem (1) is a monotone submodular function maximization problem under the constraint represented by $\mathcal{I}$. For a suitable constraint, we can approximately solve problem (1) by evaluating a polynomial number of expectations. Examples of this class of problems include the influence maximization problem $[5,6]$, randomwalk domination problem [7], budget allocation problem [4, 8], stochastic packing problem [9], and stochastic covering problem [10].

In this study, however, we are interested in reducing risk instead of maximizing expected utility. Let us consider the following example.

Example 1.1. The influence maximization problem [6] is a standard model of information spread over a network. Here, we consider the independent cascading model defined as follows: Let $G=(V, E)$ be a directed or undirected graph with vertex set $V$ and edge set $E$, and let $p: E \rightarrow[0,1]$ be an edge weight function. Each vertex has two states, active or inactive; initially, all vertices are inactive. The "information" spreads over G via the following stochastic process: When vertex $u$ becomes active at the $t$-th step, it has a single chance of activating each inactive neighbor $v$, i.e., vertex $v$ becomes active at the $t+1$-th step with probability $p(u, v)$. The problem then is to maximize the expected number of active vertices by selecting subset $X \subseteq V$ of size $k$ that is activated at the first step. Note that this problem is often considered in the area of viral marketing for finding good "influencers" in a social network.

The above problem is an expected utility maximization problem, i.e., problem (1). We have the following: Let $\Omega$ be the family of subgraphs of $G$. The probability of drawing subgraph $\omega \in \Omega$ is given by

$$
\mathbf{P}\{\omega \in \Omega\}=\prod_{e \in E(\omega)} p(e) \prod_{e \in E \backslash E(\omega)}(1-p(e)),
$$

where $\mathbf{P}$ denotes the probability, and $E(\omega)$ denotes the edges of graph $\omega$. In other words, each $e \in E$ independently appears in graph $\omega$ with probability $p(e)$. Furthermore, let $f(X, \omega)$ be the number of vertices reachable from $X$. Then, $f(X, \omega)$ is a monotone submodular function in $X$. The expected number of active vertices is then equal to $\mathbf{E}[f(X, \omega)]$. 


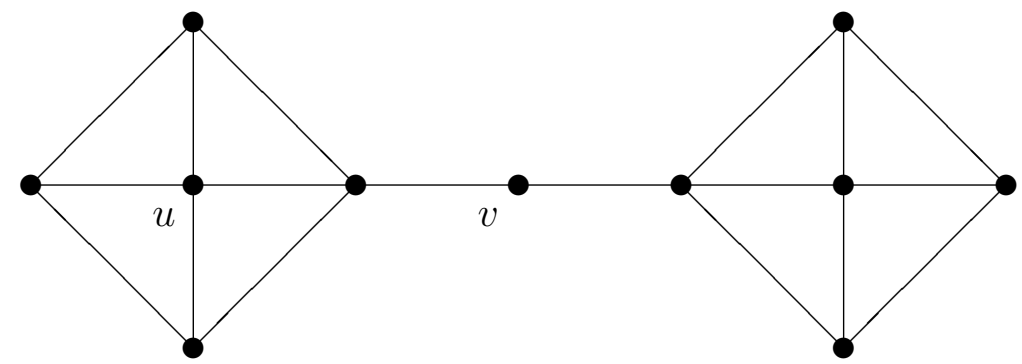

Figure 1: For an influence maximization problem, vertex $u$ is considered a "low-risk and low return" seed, whereas vertex $v$ is a "high-risk and high-return" seed.

Next, we reconsider this problem in terms of risk. Consider the graph shown in Figure 1. If vertex $u$ is the seed, the left vertices surrounding $u$ become active with high probability. On the other hand, if vertex $v$ is the seed, both left and right vertices may be activated; however, the risk of both vertices remaining inactive is considerable. Thus, we say that u is a "low-risk and low-return" seed, whereas $v$ is a "high-risk and high-return" seed. Which vertex is preferred for the seed?

In many applications, particularly in finance and health, risk is regarded as undesirable; this preference is called risk aversion [11]. In this study, we consider the computational complexity for finding a risk-averse solution to stochastic submodular utility maximization problems.

\section{Contributions}

This study makes the following contributions:

- We adopt value-at-risk (VaR) and conditional value-at-risk (CVaR) [12, 13] to measure the risk of stochastic submodular utility maximization problems. Unfortunately, VaR and CVaR are not necessarily submodular; however, CVaR is representable as the maximum of submodular functions parameterized by a smooth parameter (Section 2).

- We consider the CVaR maximization problem to find a risk-averse solution. We show the following hardness results: For confidence level $\alpha>1-1 / e$, the problem is hard to approximate within any multiplicative factor. Moreover, for confidence level $\alpha>0$ and any $\beta>0$ with $(1-\alpha)(1-\beta)<1 / e$, the problem is hard to approximate within factor $\beta$ (Section 3). 


\section{Value-at-risk and conditional value-at-risk}

In the stochastic utility maximization problem, risk is the possibility of obtaining a much smaller than expected utility. We quantify risk by two risk measures, VaR and CVaR. Among several risk measures [14] (e.g., variance), VaR and CVaR are most commonly used in many areas because they are downside risk measures (i.e., "less than the expectation" is a risk, but "greater than the expectation" is not a risk) and have some desired properties for risk measures, as discussed in $[15,16]$. In this study, we apply VaR and CVaR to stochastic submodular utility functions.

Let $f: 2^{V} \times \Omega \rightarrow \mathbb{R}$ be monotone submodular in the first variable and integrable in the second variable. Let $\alpha \in(0,1)$ be a confidence level, usually set to $\alpha=0.95$ or 0.99 [15]. The VaR at confidence level $\alpha$ is defined as

$$
\operatorname{VaR}_{\alpha}(X):=\max \{t: P\{f(X, \omega) \geq t\} \geq \alpha\} \text {. }
$$

Here, VaR guarantees that utility $f(X, \omega)$ is greater than or equal to $\operatorname{VaR}_{\alpha}(X)$ with high probability (i.e., at least $\alpha$ ). Although $\mathrm{VaR}$ is naturally defined, CVaR is more appropriate from both theoretical and practical perspectives. Let $F_{\alpha}: 2^{V} \times \mathbb{R}$ be an auxiliary function defined as

$$
F_{\alpha}(X, t)=-\frac{\alpha}{1-\alpha} t+\frac{1}{1-\alpha} \mathbf{E}[\min \{f(X, \omega), t\}] .
$$

Then, the CVaR at confidence level $\alpha$ is defined by

$$
\mathrm{CVaR}_{\alpha}(X):=\max _{t} F_{\alpha}(X, t)
$$

By definition, both $\operatorname{VaR}_{\alpha}(X)$ and $\operatorname{CVaR}_{\alpha}(X)$ are nonnegative monotone functions. The following properties of $\mathrm{VaR}$ and $\mathrm{CVaR}$ are well-known in the continuous case [13] and hold in the discrete case, since the same proofs in [13] work in both cases; thus, we omit the proofs here.

Proposition 2.1. The maximum in (4) is attained at $t=\operatorname{VaR}_{\alpha}(X)$.

Proposition 2.2. VaR and CVaR are related by

$\mathbf{E}\left[f(X, \omega) \mid f(X, \omega)<\operatorname{VaR}_{\alpha}(X)\right] \leq \operatorname{CVaR}_{\alpha}(X) \leq \mathbf{E}\left[f(X, \omega) \mid f(X, \omega) \leq \operatorname{VaR}_{\alpha}(X)\right]$. 


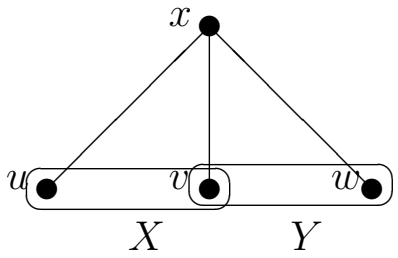

\begin{tabular}{c|ccc}
\hline$S$ & $\mathbf{E}[f(S, \omega)]$ & $\mathrm{VaR}_{\alpha}(S)$ & $\mathrm{CVaR}_{\alpha}(S)$ \\
\hline$X$ & 3.125 & 2 & 2 \\
$Y$ & 3.125 & 2 & 2 \\
$X \cap Y$ & 2 & 1 & 1 \\
$X \cup Y$ & 3.875 & 4 & 3.375 \\
\hline
\end{tabular}

Figure 2: Example of non-submodularity of VaR and CVaR in the influence maximization problem. Here, all edge weights are $p(e)=1 / 2$. Let $\alpha=4 / 5$. By setting $X=\{u, v\}$ and $Y=\{v, w\}$, we observe the non-submodularity of VaR and CVaR.

Proposition 2.2 gives an intuitive description of $\mathrm{CVaR}$, i.e., it is the conditional expectation of utility below $\operatorname{VaR}$. This implies $\operatorname{CVaR}_{\alpha}(X) \leq \operatorname{VaR}_{\alpha}(X)$, i.e., CVaR is a more conservative measure than VaR.

Remark 2.3. VaR and CVaR are usually defined for a loss function and therefore minimized. Thus our versions are the opposite of standard definitions. This is therefore sometimes called the (conditional) value-at-risk of profit [17].

In the continuous case, if a utility function is concave, CVaR is also concave; however, in the discrete case, even if utility function $f$ is monotone submodular, VaR and CVaR are not necessarily submodular. We verify this by the counterexample shown in Figure 2.

Here, VaR and CVaR are not necessarily submodular; however, CVaR still seems more tractable than VaR because CVaR is representable as the maximum of submodular functions that are parametrized by a smooth parameter, i.e., auxiliary function $F_{\alpha}(X, t)$ is submodular in $X$ and concave in $t$.

Proposition 2.4. $F_{\alpha}(X, t)$ is concave in $t$ and monotone submodular in $X$.

Proof. Since $\min \{f(X, \omega), t\}$ is concave in $t$, its expectation is also concave in $t$. Similarly, if $f(X, \omega)$ is monotone submodular in $X, \min \{f(X, \omega), t\}$ is also monotone submodular in $X$, and hence its expectation is also monotone submodular in $X$.

Note that $F_{\alpha}(X, t)$ can be negative, i.e., $F_{\alpha}(\emptyset, t)=-\alpha t /(1-\alpha)<0$ for $t>0$.

Remark 2.5. In the continuous case, if stochastic utility function $f(x, \omega)$ is concave in $x$, the corresponding auxiliary function $F_{\alpha}(x, t)$ is jointly concave 
in $(x, t)$. Therefore, $\mathrm{CVaR}_{\alpha}(x)=\min _{t} F_{\alpha}(x, t)$ is concave in $x$. In contrast, in the discrete case, $F_{\alpha}(X, t)$ is a "mixed function" (i.e., discrete in $X$ and continuous in $t)$; hence, the similar property does not hold here.

\section{Hardness of CVaR maximization problems}

To find a risk-averse solution, we consider the following CVaR maximization problem:

$$
\text { maximize } \operatorname{CVaR}_{\alpha}(X) \text { subject to } X \in \mathcal{I} \text {. }
$$

We show the following hardness results, which are the main results of this study.

Proposition 3.1. (a) Unless $\mathrm{P}=\mathrm{NP}$, there exists a constant $\bar{\alpha}>0$ such that for all $\alpha>\bar{\alpha}$, there is no polynomial time multiplicative approximation algorithm for problem (6) even for $\mathcal{I}=\{X \subseteq V:|X| \leq k\}$.

(b) Unless $\mathrm{NP} \subseteq \operatorname{DTIME}\left(n^{\log \log n}\right)$, for all $\alpha>1-1 /$ e, there is no polynomial time multiplicative approximation algorithm for problem (6) even for $\mathcal{I}=$ $\{X \subseteq V:|X| \leq k\}$.

(c) Unless $\mathrm{NP} \subseteq \mathrm{DTIME}\left(n^{\log \log n}\right)$, there is no polynomial time $\beta$ approximation algorithm for problem $(6)$ if $(1-\alpha)(1-\beta) \leq 1 /$ e, even for $\mathcal{I}=\{X \subseteq V:|X| \leq k\}$.

We prove these results below by a reduction from Minimum Dominating Set problem:

Problem 3.2 (Minimum Dominating Set). Let $G=(V, E)$ be an undirected graph with $|V|=n$. Find a smallest subset $X \subseteq V$ such that $N(X)=V$, where $N(X)=X \cup\{v \in V:(u, v) \in E\}$ is the neighbor of $X$.

Minimum Dominating Set problem admits the following hardness results. Note that These results are originally stated for Set Cover problem; however, this problem is equivalent to Minimum Dominating Set problem, and is more convenient for our use here.

Theorem 3.3 (Raz and Safra [18]). Unless $\mathrm{P}=\mathrm{NP}$, for some constant $c>$ 0 , there is no polynomial time $c \log n$ approximation algorithm for Minimum Dominating Set problem. 
Theorem 3.4 (Feige [19]). Unless NP $\subseteq \operatorname{DTIME}\left(n^{\log \log n}\right)$, for any $\epsilon>0$, there is no polynomial time $(1-\epsilon) \log n$ approximation algorithm for Minimum Dominating Set problem.

Let $G=(V, E)$ be an instance of Minimum Dominating Set problem. We then consider the following instance of the VaR/CVaR maximization problem: Let $\Omega=V$; utility function $f: 2^{V} \times \Omega \rightarrow \mathbb{R}$ is then defined as

$$
f(X, \omega)= \begin{cases}1, & \omega \in N(X), \\ 0, & \text { otherwise }\end{cases}
$$

which is monotone submodular in $X$. Let $\mathcal{I}=\{X \subseteq V:|X| \leq k\}$.

We consider the following two cases, $\alpha=1$ and $\alpha<1$.

Case $1(\alpha=1)$. In this case, $\operatorname{VaR}_{\alpha}(X)=\operatorname{CVaR}_{\alpha}(X)=1$ if $X$ is a dominating set, otherwise 0 . Therefore $\max _{X \in \mathcal{I}} \operatorname{VaR}_{\alpha}(X)=\max _{X \in \mathcal{I}} \mathrm{CVaR}_{\alpha}(X)>0$ if and only if there exists a dominating set of size $k$. This implies that, if there are multiplicative approximation algorithms for the VaR or CVaR maximization problem, it can be used to solve Minimum Dominating Set problem. This shows all results for the case of $\alpha=1$.

Case $2(\alpha<1)$. By definition of $\operatorname{VaR}_{\alpha}$, we have

$$
\operatorname{VaR}_{\alpha}(X)= \begin{cases}1, & \operatorname{dom}(X) \geq \alpha n \\ 0, & \text { otherwise }\end{cases}
$$

where $\operatorname{dom}(X)$ is the number of vertices dominated by $X$. By Proposition 2.1, we have

$$
\operatorname{CVaR}_{\alpha}(X)=F(X, 0)=0
$$

for $\operatorname{dom}(X)<\alpha n$, and

$$
\begin{aligned}
\operatorname{CVaR}_{\alpha}(X) & =F(X, 1) \\
& =\frac{-\alpha}{1-\alpha}+\frac{\mathbf{E}[f(X, \omega)\}]}{1-\alpha} \\
& =\frac{\operatorname{dom}(X) / n-\alpha}{1-\alpha}
\end{aligned}
$$


for $\operatorname{dom}(X) \geq \alpha n$. Therefore

$$
\mathrm{CVaR}_{\alpha}(X)=\max \left\{\frac{\operatorname{dom}(X) / n-\alpha}{1-\alpha}, 0\right\} .
$$

Suppose that there is a $\beta$ approximation algorithm for $\max _{|X| \leq k} \operatorname{CVaR}_{\alpha}(X)$. For an integer $k$, we find $\beta$ approximate solution $X$ to $\max _{|X| \leq k} \operatorname{CVaR}_{\alpha}(X)$. If $\mathrm{CVaR}_{\alpha}(X)<\beta$, we terminate this procedure. Otherwise, we remove all vertices dominated by $X$ from $G$ and repeat this procedure. If all vertices are removed, we output the union of $X \mathrm{~s}$ as an approximate solution to Minimum Dominating Set problem. We perform the above procedure for all $k=1, \ldots, n$ to find the minimum $k$ such that the procedure gives a solution.

Analyzing this procedure, we first let $k^{*}$ be the size of the minimum dominating set. Then we have $k \leq k^{*}$ since $\max _{|X| \leq k^{*}} \operatorname{CVaR}_{\alpha}(X)=1$ by (11); hence, $\operatorname{CVaR}_{\alpha}(X) \geq \beta$ for $k=k^{*}$. Next, we evaluate the number of iterations $T$. When $\operatorname{CVaR}_{\alpha}(X) \geq \beta$, we have $\operatorname{dom}(X) \geq(\alpha+\beta-\alpha \beta) n$ by (11). Thus, after one iteration, we have a smaller graph that has at most $(1-\alpha)(1-\beta) n$ vertices with a dominating set of size at most $k^{*}$. Therefore, by solving $(1-\alpha)^{T}(1-\beta)^{T} n \leq 1$, we have $T \leq \log n / \log (1 /(1-\alpha)(1-\beta))$.

The output size is at most $k T \leq k^{*} \log n / \log (1 /(1-\alpha)(1-\beta))$. If $(1-\alpha)(1-\beta)<1 / e$, it is a $(1-\epsilon) \log n$ approximation algorithm to Minimum Dominating Set problem; therefore, by Theorem 3.4, we have Proposition 3.1 (c).

Since this proof is easily modified to prove Propositions 3.1 (a) and 3.1 (b), we omit these proofs.

Remark 3.5. In the continuous case, confidence level $\alpha$ is usually set to $\alpha=0.95$ or 0.99 [15]; however, Proposition 3.1 shows that such high $\alpha$ is computationally intractable in the discrete case.

\section{Related work}

Risk-averse solutions in the continuous case have been extensively studied in the literature $[12,13,20]$; however, risk-averse solutions in a discrete setting have been largely overlooked.

Ishii et al. [21] studied the stochastic spanning tree problem that minimizes VaR in which edge weights followed normal distributions. Goel and Indyk [22] considered the bin packing and knapsack problems with a VaR constraint in which item sizes followed specific distributions. Atamtürk and 
Narayanan [23] discussed the risk-averse portfolio selection problem under a polymatroid constraint in which item prices followed normal distributions. The key finding of these three studies was that VaR could be expressed in a closed form in these settings.

Unlike these studies, our study assumes no specific probability distribution. Hence, our method is applicable to the influence maximization, randomwalk domination, and various other problems that have recently gained much interest in the machine learning community.

Krause, McMahan, and Guestrin [24] introduced the robust submodular maximization problem, which maximizes $f(X):=\min \left\{f_{1}(X), \ldots, f_{m}(X)\right\}$ for multiple objective functions $f_{1}, \ldots, f_{m}$. In our formulation, as $\alpha \rightarrow 1$, we have $\operatorname{VaR}_{\alpha}(X)=\operatorname{CVaR}_{\alpha}(X)=\min \{f(X, \omega): \omega \in \Omega\}$. Therefore the formulation proposed in [24] is a special case of our VaR/CVaR formulation.

Very recently, Zhang et al. [25] considered the seed set minimization problem, which is very similar to our setting. Their problem was to find the smallest subset $X$ that yields $\operatorname{VaR}_{\alpha}(X) \geq \theta$ for a given threshold $\theta$. They showed that a greedy solution for the expected utility has a theoretical guarantee for this problem.

\section{Acknowledgement}

This work was supported by JST, ERATO, Kawarabayashi Large Graph Project.

\section{References}

[1] B. Lehmann, D. Lehmann, N. Nisan, Combinatorial auctions with decreasing marginal utilities, in: Proceedings of the 3rd ACM Conference on Electronic Commerce, 2001, pp. 18-28.

[2] G. L. Nemhauser, L. A. Wolsey, M. L. Fisher, An analysis of approximations for maximizing submodular set function i, Mathematical Programming 14 (1) (1978) 265-294.

[3] G. Calinescu, C. Chekuri, M. Pál, J. Vondrák, Maximizing a monotone submodular function subject to a matroid constraint, SIAM Journal on Computing 40 (6) (2011) 1740-1766. 
[4] M. Sviridenko, A note on maximizing a submodular set function subject to a knapsack constraint, Operations Research Letters 32 (1) (2004) 4143.

[5] P. Domingos, M. Richardson, Mining the network value of customers, in: Proceedings of the 7th ACM SIGKDD International Conference on Knowledge Discovery and Data Mining, 2001, pp. 57-66.

[6] D. Kempe, J. Kleinberg, É. Tardos, Maximizing the spread of influence through a social network, in: Proceedings of the 9th ACM SIGKDD International Conference on Knowledge Discovery and Data Mining, 2003, pp. 137-146.

[7] R.-H. Li, J. X. Yu, X. Huang, H. Cheng, Random-walk domination in large graphs, in: Proceedings of the 30th IEEE International Conference on Data Engineering, 2014.

[8] N. Alon, I. Gamzu, M. Tennenholtz, Optimizing budget allocation among channels and influencers, in: Proceedings of the 21st World Wide Web Conference, 2012, pp. 381-388.

[9] B. C. Dean, M. X. Goemans, J. Vondrák, Approximating the stochastic knapsack problem: The benefit of adaptivity, in: Proceedings of the 45th Annual IEEE Symposium on Foundations of Computer Science, 2004, pp. 208-217.

[10] M. Goemans, J. Vondrák, Stochastic covering and adaptivity, in: Proceedings of the 7th Latin American Theoretical Informatics, 2006, pp. $532-543$.

[11] J. W. Pratt, Risk aversion in the small and in the large, Econometrica: Journal of the Econometric Society (1964) 122-136.

[12] R. T. Rockafellar, S. Uryasev, Optimization of conditional value-at-risk, Journal of risk 2 (2000) 21-42.

[13] R. T. Rockafellar, S. Uryasev, Conditional value-at-risk for general loss distributions, Journal of Banking \& Finance 26 (7) (2002) 1443-1471.

[14] C. Alexander, Market Risk Analysis, Value at Risk Models, Vol. 4, John Wiley \& Sons, 2009. 
[15] Bank for International Settlements, Fundamental review of the trading book - second consultative document, retrieved at October 2013 (2013). URL http: //www.bis.org/publ/bcbs219.htm

[16] R. Rockafellar, J. Royset, S. Miranda, Superquantile regression with applications to buffered reliability, uncertainty quantification, and conditional value-at-risk, European Journal of Operational Research 234 (1) (2014) 140-154.

[17] J. Calatrava, A. Garrido, Spot water markets and risk in water supply, Agricultural economics 33 (2) (2005) 131-143.

[18] R. Raz, S. Safra, A sub-constant error-probability low-degree test, and a sub-constant error-probability pcp characterization of np, in: Proceedings of the 29th Annual ACM Symposium on Theory of Computing, 1997, pp. 475-484.

[19] U. Feige, A threshold of $\ln n$ for approximating set cover, Journal of the ACM 45 (4) (1998) 634-652.

[20] S. Ahmed, Convexity and decomposition of mean-risk stochastic programs, Mathematical Programming 106 (3) (2006) 433-446.

[21] H. Ishii, S. Shiode, T. Nishida, Y. Namasuya, Stochastic spanning tree problem, Discrete Applied Mathematics 3 (4) (1981) 263-273.

[22] A. Goel, P. Indyk, Stochastic load balancing and related problems, in: Proceedings of the 40th Annual IEEE Symposium on Foundations of Computer Science, 1999, pp. 579-586.

[23] A. Atamtürk, V. Narayanan, Polymatroids and mean-risk minimization in discrete optimization, Operations Research Letters 36 (5) (2008) 618622 .

[24] A. Krause, H. B. McMahan, C. Guestrin, A. Gupta, Robust submodular observation selection, Journal of Machine Learning Research 9 (12).

[25] P. Zhang, W. Chen, X. Sun, Y. Wang, J. Zhang, Minimizing seed set selection with probabilistic coverage guarantee in a social network, in: Proceedings of the 20th ACM SIGKDD international conference on Knowledge discovery and data mining, ACM, 2014, pp. 1306-1315. 